\title{
LOG-CONCAVITY OF THE DUISTERMAAT-HECKMAN MEASURE FOR SEMIFREE HAMILTONIAN $S^{1}$-ACTIONS
}

\author{
YUNHYUNG CHO \\ (Communicated by Lei $\mathrm{Ni}$ ) \\ This paper is dedicated to my father
}

\begin{abstract}
The Ginzberg-Knutson conjecture states that for any Hamiltonian Lie group $G$-action, the corresponding Duistermaat-Heckman measure is logconcave. It turns out that the conjecture is not true in general, but every wellknown counterexample has non-isolated fixed points. In this paper, we prove that if the Hamiltonian circle action on a compact symplectic manifold $(M, \omega)$ is semifree and all fixed points are isolated, then the Duistermaat-Heckman measure is log-concave. With the same assumption, we also prove that $\omega$ and every reduced symplectic form satisfy the hard Lefschetz property.
\end{abstract}

\section{INTRODUCTION}

In statistical mechanics, consider the relation $S(E)=k \log W(E)$, which is called Boltzmann's principle, where $W(E)$ is the number of states with given values of macroscopic parameters $E$ (like energy, temperature, $\cdots$ ), $k$ is the Boltzmann's constant, and $S$ is the entropy of the system which measures the degree of disorder in the system. For the additive values $E$, it is well-known that the entropy is always a concave function. (See [18] for more details.)

Now, consider a Hamiltonian $G$-manifold $(M, \omega)$ with the moment map $\mu: M \rightarrow$ $\mathfrak{g}^{*}$. The Liouville measure $m_{L}$ is defined by

$$
m_{L}(U):=\int_{U} \frac{\omega^{n}}{n !}
$$

for any open set $U \subset M$. The push-forward measure $m_{\mathrm{DH}}:=\mu_{*} m_{L}$ is called the Duistermaat-Heckman measure. Then $m_{\mathrm{DH}}$ can be regarded as a measure on $\mathfrak{g}^{*}$ such that for any Borel subset $B \subset \mathfrak{g}^{*}, m_{\mathrm{DH}}(B)=\int_{\mu^{-1}(B)} \frac{\omega^{n}}{n !}$ tells us how many states of our system have momenta in $B$. By the Duistermaat-Heckman theorem [5], $m_{\mathrm{DH}}$ can be expressed in terms of the density function $\mathrm{DH}(\xi)$ with respect to the Lebesque measure on $\mathfrak{g}^{*}$. Hence if we consider Boltzmann's principle in our Hamiltonian system with an identification $W=\mathrm{DH}$, it is natural to ask whether the Duistermaat-Heckman measure $m_{\mathrm{DH}}$ is log-concave. As noted in [17, [1], and [14, V. Ginzburg and A. Knutson conjectured that for any closed Hamiltonian $T$-manifold, the corresponding Duistermaat-Heckman measure is log-concave.

Received by the editors July 22, 2012.

2010 Mathematics Subject Classification. Primary 37J05, 53D20; Secondary 37J10.

Key words and phrases. Symplectic geometry, Duistermaat-Heckman measure. 
The log-concavity problem of the Duistermaat-Heckman measure is proved by A. Okounkov [16] when $M$ is a co-adjoint orbit of the classical Lie groups of type $A_{n}, B_{n}$, or $C_{n}$ with the maximal torus action. Around the same time, W. Graham [7. proved that the log-concavity property holds for any holomorphic Hamiltonian circle action on any Kähler manifold. But the counterexample was found by Y. Karshon 11. By using Lerman's symplectic cutting method, she constructed a closed 6-dimensional semifree Hamiltonian $S^{1}$-manifold with two fixed components such that the Duistermaat-Heckman measure is not log-concave. Later, Y. Lin 14 generalized the construction of 6-dimensional Hamiltonian $S^{1}$-manifolds which do not satisfy the log-concavity of the Duistermaat-Heckman measure. But all counterexamples of Karshon and Lin are the cases when each fixed component is of codimension two (i.e. non-isolated). So, the log-concavity problem is still open for the case when $(M, \omega)$ is a Hamiltonian $S^{1}$-manifold whose fixed components are of codimension greater than two. In this paper, we will show that

Theorem 1.1. Let $(M, \omega)$ be a closed symplectic manifold with a semifree Hamiltonian $S^{1}$-action whose fixed point set $M^{S^{1}}$ consists of isolated points. Then the Duistermaat-Heckman measure is log-concave.

The conditions "semifree" and "isolated fixed points" enable us to use the Tolman-Weitsman basis [19] of the equivariant cohomology $H_{S^{1}}^{*}(M)$. As you will see in Section 2, any semifree Hamiltonian $S^{1}$-manifold with only isolated fixed points has a lot of remarkable properties. In fact, the cohomology ring and the equivariant cohomology ring of $M$ are the same as those of $S^{2} \times \cdots \times S^{2}$ with a diagonal semifree circle action. In particular, $(M, \omega)$ is equivariantly symplectomorphic to the product space of $S^{2}$ copies $\left(S^{2} \times \cdots \times S^{2}, \sigma\right)$ with some $S^{1}$-invariant Kähler structure $\sigma$ when $\operatorname{dim} M \leq 6$. (See [12] and [6].) Therefore, we may ask whether $(M, \omega)$ satisfies the properties which the diagonal circle action on $\left(S^{2} \times \cdots \times S^{2}, \sigma\right)$ satisfies. In this paper, we prove the following.

Theorem 1.2. Let $(M, \omega)$ be a closed semifree Hamiltonian $S^{1}$-manifold whose fixed points are all isolated, and let $\mu$ be the moment map. Then $\omega$ satisfies the hard Lefschetz property. Moreover, the reduced symplectic form $\omega_{t}$ satisfies the hard Lefschetz property for every regular value $t$.

In Section 2 we briefly review Tolman and Weitsman's work 19 which is very powerful to analyze the equivariant cohomology of the Hamiltonian $S^{1}$-manifold with isolated fixed points as we mentioned above. Especially we use the TolmanWeitsman basis of the equivariant cohomology $H_{S^{1}}^{*}(M)$ which is constructed by using the equivariant version of Morse theory [20]. In Section 3, we express the Duistermaat-Heckman function explicitly in terms of the integration of some cohomology class on the reduced space. Then we compute the integration by using the Kirwan-Jeffrey residue formula [10. Consequently, we will show that the logconcavity of the Duistermaat-Heckman measure is completely determined by the set of pairs $\left\{\left(\mu(F), m_{F}\right)_{F} \mid F \in M^{S^{1}}\right\}$, where $\mu(F)$ is the image of the moment map of $F$ and $m_{F}$ is the product of all weights of the $S^{1}$-representation on $T_{F} M$ (Proposition 3.9). In Section 4, we will prove Theorem 1.1, and we will prove Theorem 1.2 in Section 5 . 


\section{TOLMAn-Weitsman Basis \\ OF THE EQUIVARIANT COHOMOLOGY $H_{S^{1}}^{*}(M ; \mathbb{Z})$}

In this section we briefly review Tolman and Weitsman's results in 19. Throughout this section, we assume that $\left(M^{2 n}, \omega\right)$ is a closed semifree Hamiltonian $S^{1}$ manifold whose fixed points are isolated. Note that for each fixed point $p \in M^{S^{1}}$, the index of $p$ is the Morse index of the moment map at $p$, which is the same as twice the number of negative weights of the tangential $S^{1}$-representation at $p$.

Proposition 2.1 ([19]). Let $N_{k}$ be the number of fixed points of index $2 k$. Then $N_{k}=\left(\begin{array}{l}n \\ k\end{array}\right)$. Hence $N_{k}$ is the same as that of the standard diagonal circle action on $\left(S^{2} \times \cdots \times S^{2}, \omega_{1} \oplus \cdots \oplus \omega_{n}\right)$, where $\omega_{i}$ is the Fubini-Study form on $S^{2}$ of $i$-th factor.

Theorem 2.2 ([19]). Let $2^{[n]}$ be the power set of $\{1, \cdots, n\}$. Then there exists a bijection $\phi: M^{S^{1}} \rightarrow 2^{[n]}$ satisfying the following:

(1) For each index-2k fixed point $x \in M^{S^{1}},|\phi(x)|=k$.

(2) Let $u$ be the generator of $H^{*}\left(B S^{1}, \mathbb{Z}\right)$. For each index-2k fixed point $x \in$ $M^{S^{1}}$, there exists a unique cohomology class $\alpha_{x} \in H_{S^{1}}^{2 k}(M ; \mathbb{Z})$ such that for any $x^{\prime} \in M^{S^{1}}$,

- $\left.\alpha_{x}\right|_{x^{\prime}}=u^{k}$ if $\phi(x) \subset \phi\left(x^{\prime}\right)$.

- $\left.\alpha_{x}\right|_{x^{\prime}}=0$ otherwise.

Here, $\left.\alpha\right|_{x^{\prime}}$ means the image $\pi_{x^{\prime}}\left(i^{*}(\alpha)\right)$ where $i^{*}: H_{S^{1}}^{*}(M ; Z) \rightarrow H_{S^{1}}^{*}\left(M^{S^{1}} ; \mathbb{Z}\right)$ is a homomorphism induced by an inclusion $i: M^{S^{1} \hookrightarrow} \hookrightarrow$, and $\pi_{x^{\prime}}: H_{S^{1}}^{*}\left(M^{S^{1}} ; \mathbb{Z}\right) \rightarrow$ $H_{S^{1}}^{*}\left(x^{\prime} ; \mathbb{Z}\right)$ is a natural projection. Moreover $\left\{\alpha_{x} \mid x \in M^{S^{1}}\right\}$ forms a basis of $H_{S^{1}}^{*}(M ; \mathbb{Z})$.

If we apply Theorem 2.2 to $\left(S^{2} \times \cdots \times S^{2}, \omega_{1} \oplus \cdots \oplus \omega_{n}\right)$ with the diagonal semifree Hamiltonian circle action, we get a bijection $\psi:\left(S^{2} \times \cdots \times S^{2}\right)^{S^{1}} \rightarrow 2^{[n]}$ and there is a basis $\left\{\beta_{y} \mid y \in\left(S^{2} \times \cdots \times S^{2}\right)^{S^{1}}\right\}$ of $H_{S^{1}}^{*}\left(S^{2} \times \cdots \times S^{2} ; \mathbb{Z}\right)$ that satisfies the conditions in Theorem 2.2. Hence we have an identification map

$$
\psi^{-1} \circ \phi: M^{S^{1}} \rightarrow\left(S^{2} \times \cdots \times S^{2}\right)^{S^{1}}
$$

and $\psi^{-1} \circ \phi$ preserves the indices of the fixed points.

Note that $\psi^{-1} \circ \phi$ gives an identification between $H_{S^{1}}^{*}(M ; \mathbb{Z})$ and $H_{S^{1}}^{*}\left(S^{2} \times \cdots \times\right.$ $\left.S^{2} ; \mathbb{Z}\right)$ as follows. Let $a_{i}=\alpha_{\phi^{-1}\{i\}} \in H_{S^{1}}^{2}(M ; \mathbb{Z})$ and $b_{i}=\beta_{\psi^{-1}\{i\}} \in H_{S^{1}}^{2}\left(S^{2} \times\right.$ $\left.\cdots \times S^{2} ; \mathbb{Z}\right)$. The following lemma is proved by Tolman and Weitsman in [19], but we give a complete proof here to use their idea in the rest of this paper.

Lemma 2.3 ([19]). For each $x \in M^{S^{1}}$, we have $\alpha_{x}=\prod_{j \in \phi(x)} a_{j}$. Similarly, we have $\beta_{y}=\prod_{j \in \psi(y)} b_{j}$ for each $y \in\left(S^{2} \times \cdots \times S^{2}\right)^{S^{1}}$.

Proof. For an inclusion $i: M^{S^{1}} \hookrightarrow M$, we have a natural ring homomorphism $i^{*}: H_{S^{1}}^{*}(M) \rightarrow H_{S^{1}}^{*}\left(M^{S^{1}}\right) \cong H^{*}\left(M^{S^{1}}\right) \otimes H *\left(B S^{1}\right)$. Kirwan's injectivity theorem [13] implies that $i^{*}$ is an injective ring homomorphism. Hence it is enough to show that $\left.\alpha_{x}\right|_{z}=\left.\left(\prod_{j \in \phi(x)} a_{j}\right)\right|_{z}$ for all $x, z \in M^{S^{1}}$. For any $x, z \in M^{S^{1}}$ with $\operatorname{Ind}(x)=2 k$,

- $\left.\alpha_{x}\right|_{z}=u^{k}$ if $\phi(x) \subset \phi(z)$.

- $\left.\alpha_{x}\right|_{z}=0$ otherwise. 
On the other hand, $\left.\left(\prod_{j \in \phi(x)} a_{j}\right)\right|_{z}=\left.\prod_{j \in \phi(x)} a_{j}\right|_{z}$. Since $\left.a_{j}\right|_{z}=u$ if and only if $j \in \phi(z)$, we have

- $\left.\left(\prod_{j \in \phi(x)} a_{j}\right)\right|_{z}=u^{k}$ if $\phi(x) \subset \phi(z)$.

- $\left.\left(\prod_{j \in \phi(x)} a_{j}\right)\right|_{z}=0$ otherwise.

Therefore, we have $\alpha_{x}=\prod_{j \in \phi(x)} a_{j}$. The proof of the second statement is similar.

Hence the $H^{*}\left(B S^{1}\right)$-module isomorphism $f: H_{S^{1}}^{*}(M ; \mathbb{Z}) \rightarrow H_{S^{1}}^{*}\left(S^{2} \times \cdots \times S^{2} ; \mathbb{Z}\right)$ which sends $\alpha_{x}$ to $\beta_{\psi^{-1} \circ \phi(x)}$ for each $x \in M^{S^{1}}$ is in fact a ring isomorphism by Lemma 2.3. To sum up, we have the following corollary.

Corollary 2.4 ([19]). There is a ring isomorphism

$$
f: H_{S^{1}}^{*}(M ; \mathbb{Z}) \rightarrow H_{S^{1}}^{*}\left(S^{2} \times \cdots \times S^{2} ; \mathbb{Z}\right)
$$

which sends $\alpha_{x}$ to $\beta_{\psi^{-1} \circ \phi(x)}$. Moreover, for any $\alpha \in H_{S^{1}}^{*}(M ; \mathbb{Z})$ and any fixed point $x \in M^{S^{1}}$, we have $\alpha_{x}=\left.f(\alpha)\right|_{\psi^{-1} \circ \phi(x)}$.

\section{The Duistermaat-Heckman function and the Residue formula}

Let $(M, \omega)$ be a $2 n$-dimensional closed Hamiltonian $S^{1}$-manifold with the moment map $\mu: M \rightarrow \mathbb{R}$. We may assume that 0 is a regular value of $\mu$ such that $\mu^{-1}(0)$ is non-empty. Choose two consecutive critical values $c_{1}$ and $c_{2}$ of $\mu$ so that the open interval $\left(c_{1}, c_{2}\right)$ consists of regular values of $\mu$ and contains 0 . By the Duistermaat-Heckman's theorem $[5],\left[\omega_{t}\right]=\left[\omega_{0}\right]-e t$ where $e$ is the Euler class of $S^{1}$-fibration $\mu^{-1}(0) \rightarrow M_{0}$, where $M_{0}$ is the symplectic reduction at 0 with the induced symplectic form $\omega_{0}$. Hence we have

$$
\mathrm{DH}(t)=\int_{M_{0}} \frac{1}{(n-1) !}\left(\left[\omega_{0}\right]-e t\right)^{n-1}
$$

on $\left(c_{1}, c_{2}\right) \subset \operatorname{Im} \mu$.

Note that a continuous function on an open interval $g:(a, b) \rightarrow \mathbb{R}$ is concave if $g(t c+(1-t) d) \geq \operatorname{tg}(c)+(1-t) g(d)$ for any $c, d \in(a, b)$ and for any $t \in(0,1)$. We remark the basic property of a concave function as follows.

Remark 3.1. Let $g$ be a continuous, piecewise smooth function on a connected interval $I \subset \mathbb{R}$. Then $g$ is concave on $I$ if and only if the derivative of $g$ is decreasing, i.e. $g^{\prime \prime}(t) \leq 0$ for every smooth point $t \in I$ and $g_{+}^{\prime}(c)-g_{-}^{\prime}(c)<0$ for every singular point $c \in I$, where $g_{+}^{\prime}(c)=\lim _{t \rightarrow c, t>c} g^{\prime}(t)$ and $g_{-}^{\prime}(c)=\lim _{t \rightarrow c, t<c} g^{\prime}(t)$.

Note that Duistermaat and Heckman proved that DH is a polynomial on a connected regular open interval $U \subset \mu(M)$. The following formula due to Guillemin, Lerman, and Sternberg describes the behavior of $\mathrm{DH}$ near the critical value of $\mu$. In particular, it implies that DH is $k$-times differentiable at a critical value $c \in \mu(M)$ if and only if $\mu^{-1}(c)$ does not contain a fixed component whose codimension is less than $4+2 k$.

Theorem $3.2([8])$. Assume that $c$ is a critical value which corresponds to the fixed components $C_{i}$ 's. Then the jump of $\mathrm{DH}(t)$ at $c$ is given by

$$
\mathrm{DH}_{+}-\mathrm{DH}_{-}=\sum_{i} \frac{\operatorname{vol}\left(C_{i}\right)}{\left(d_{i}-1\right) ! \prod_{j} w_{j}}(t-c)^{d_{i}-1}+O\left((t-c)^{d_{i}}\right)
$$


where the sum is over the components $C_{i}$ of $M^{S^{1}} \cap \mu^{-1}(c), d_{i}$ is half the real codimension of $C_{i}$ in $M$, and the $w_{j}$ 's are the weights of the $S^{1}$-representation on the normal bundle of $C_{i}$.

If $c$ is a critical value which is not an extremum, then the codimension of the fixed point set in $\mu^{-1}(c)$ is at least 4 . Therefore Theorem 3.2 implies that $\mathrm{DH}(t)$ is continuous at non-extremal critical values and $\mathrm{DH}^{\prime}(t)$ jumps at $c$ when $d$ equals 2 . In the case when $d=2$, the two non-zero weights must have opposite signs, so the jump in the derivative is negative, i.e. $\mathrm{DH}^{\prime}(t)$ decreases when it passes through the critical value with $d=2$. Since $\mathrm{DH}$ is continuous, the jump in $\frac{d}{d t} \ln \mathrm{DH}(t)$ is negative at $c$. Combining with Remark 3.1, we have the following corollary.

Corollary 3.3. Let $(M, \omega)$ be a closed Hamiltonian $S^{1}$-manifold with the moment map $\mu: M \rightarrow \mathbb{R}$. Then the corresponding Duistermaat-Heckman function $\mathrm{DH}$ is log-concave on $\mu(M)$ if $(\log D H(t))^{\prime \prime} \leq 0$ for every regular value $t \in \mu(M)$.

Note that $(\log \mathrm{DH}(t))^{\prime \prime}=\frac{\mathrm{DH}(t) \cdot \mathrm{DH}^{\prime \prime}(t)-\mathrm{DH}(t)^{2}}{\mathrm{DH}(t)^{2}}$. Therefore $(\log \mathrm{DH}(t))^{\prime \prime} \leq 0$ is equivalent to $\mathrm{DH}(t) \cdot \mathrm{DH}^{\prime \prime}(t)-\mathrm{DH}^{\prime}(t)^{2} \leq 0$. Equation (3.1) implies that

$$
\mathrm{DH}(t) \cdot \mathrm{DH}^{\prime \prime}(t)=(n-1)(n-2) \int_{M_{0}} e^{2}\left[\omega_{t}\right]^{n-3} \cdot \int_{M_{0}}\left[\omega_{t}\right]^{n-1}
$$

and

$$
\mathrm{DH}^{\prime}(t)^{2}=(n-1)^{2}\left(\int_{M_{0}} e\left[\omega_{t}\right]^{n-2}\right)^{2} .
$$

To compute the integrals appearing in equations (3.2) and (3.3), we need the following procedures. For an inclusion $i: \mu^{-1}(0) \hookrightarrow M$, we have a ring homomorphism $\kappa: H_{S^{1}}^{*}(M ; \mathbb{R}) \rightarrow H_{S^{1}}^{*}\left(\mu^{-1}(0) ; \mathbb{R}\right) \cong H^{*}\left(M_{0} ; \mathbb{R}\right)$ which is called the Kirwan map. Due to the Kirwan surjectivity [13, $\kappa$ is a ring surjection. Now, consider a 2 -form $\widetilde{\omega}:=\omega-d(\mu \theta)$ on $M \times E S^{1}$ where $\theta$ is the pull-back of the connection form on $E S^{1}$ along the projection $M \times E S^{1} \rightarrow E S^{1}$. We denote $x=\pi^{*} u \in H_{S^{1}}^{2}(M ; \mathbb{Z})$ where $\pi: M \times_{S^{1}} E S^{1} \rightarrow B S^{1}$ and $u$ is a generator of $H^{*}\left(B S^{1} ; \mathbb{Z}\right)$ such that the Euler class of the Hopf bundle $E S^{1} \rightarrow B S^{1}$ is $-u$. Some part of the following two lemmas is given in 1, but we give the complete proofs here.

Lemma 3.4. $\widetilde{\omega}$ is $S^{1}$-invariant and closed, and $i_{X} \widetilde{\omega}=0$ so that $\widetilde{\omega}$ represents a cohomology class in $H_{S^{1}}^{*}(M ; \mathbb{R})$. Moreover, for any fixed component $F \in M^{S^{1}}$, we have $\kappa([\widetilde{\omega}])=\left[\omega_{0}\right]$ and $\left.[\widetilde{\omega}]\right|_{F}=\left.[\omega]\right|_{F}+\mu(F) u$. In particular, if $F$ is isolated, then $\left.[\widetilde{\omega}]\right|_{F}=\mu(F) u$.

Proof. For the first statement, it is enough to show that $i_{X} \widetilde{\omega}$ and $L_{X} \widetilde{\omega}$ vanish. Note that $i_{X} \widetilde{\omega}=i_{X} \omega-i_{X} d(\mu \theta)=-d \mu+d i_{X}(\mu \theta)-L_{X}(\mu \theta)$ by Cartan's formula. Since $i_{X}(\mu \theta)=\mu$ and $\mu \theta$ is invariant under the circle action, we have $i_{X} \widetilde{\omega}=-d \mu+d \mu=0$. Moreover, it is obvious that $\widetilde{\omega}$ is closed by definition. Hence $L_{X} \widetilde{\omega}=0$ by Cartan's formula again.

To prove the second statement, consider the following diagram:

$$
\begin{array}{cccc}
\mu^{-1}(0) \times E S^{1} & \hookrightarrow & M \times E S^{1} \\
\downarrow & & \downarrow \\
\mu^{-1}(0) \times_{S^{1}} E S^{1} & \hookrightarrow & M \times_{S^{1}} E S^{1} \\
\downarrow & & \\
\mu^{-1}(0) / S^{1} \cong M_{\text {red }} & & &
\end{array}
$$


Since $d \mu$ is zero on the tangent bundle $\mu^{-1}(0) \times E S^{1}$, the pull-back of $\widetilde{\omega}=\omega-d \mu \wedge$ $\theta-\mu d \theta$ to $\mu^{-1}(0) \times E S^{1}$ is the restriction $\left.\omega\right|_{\mu^{-1}(0) \times E S^{1}}$ and the push-forward of $\left.\omega\right|_{\mu^{-1}(0) \times E S^{1}}$ to $\mu^{-1}(0) / S^{1}$ is just a reduced symplectic form at the level 0 . Hence $\kappa([\widetilde{\omega}])=\left[\omega_{0}\right]$.

To show the last statement, consider $\left.[\widetilde{\omega}]\right|_{F}=\left.[\omega-d \mu \wedge \theta-\mu d \theta]\right|_{F}$. Since the restriction $\left.d \mu\right|_{F \times E S^{1}}$ vanishes, we have $\left.[\widetilde{\omega}]\right|_{F}=\left.[\omega]\right|_{F}-\left.\mu(F) \cdot[d \theta]\right|_{E S^{1}}=\left.[\omega]\right|_{F}+$ $\mu(F) u$. In particular, if $F$ is isolated, then we have $\left.[\widetilde{\omega}]\right|_{F}=\mu(F) u$.

Lemma 3.5. Consider a 2-form $d \theta$ on $M \times E S^{1}$, where $\theta$ is the pull-back of the connection form on $E S^{1}$ along the projection $M \times E S^{1} \rightarrow E S^{1}$. Then we can push $d \theta$ forward to $M \times{ }_{S^{1}} E S^{1}$ so that $d \theta$ represents a cohomology class in $H_{S^{1}}^{*}(M ; \mathbb{R})$. Moreover, $[d \theta]=-x$ and $\kappa([d \theta])=-\kappa(x)=e$, where $e$ is the Euler class of the $S^{1}$-fibration $\mu^{-1}(0) \rightarrow M_{\text {red }}$.

Proof. Note that $i_{X} d \theta=L_{X} \theta-d i_{X} \theta=0$. Hence we can push $d \theta$ forward to $M \times{ }_{S^{1}} E S^{1}$. For any fixed point $p \in M^{S^{1}}$, the restriction $\left.[d \theta]\right|_{p}$ is the Euler class of $p \times E S^{1} \rightarrow B S^{1}$. Hence $[d \theta]=-u \cdot 1=-x$. Here, the multiplication "." comes from the $H^{*}\left(B S^{1}\right)$-module structure on $H_{S^{1}}^{*}(M)$. By the diagram in the proof of Lemma 3.4, $\kappa([d \theta])$ is just the Euler class of the $S^{1}$-fibration $\mu^{-1}(0) \rightarrow \mu^{-1}(0) / S^{1}$. Therefore $\kappa([d \theta])=-\kappa(x)=e$.

Combining equations (3.2), (3.3), Lemma 3.4 and Lemma 3.5, we have the following corollary.

Corollary 3.6. $\mathrm{DH}(0) \cdot \mathrm{DH}^{\prime \prime}(0)-\mathrm{DH}^{\prime}(0)^{2} \leq 0$ if and only if

$$
(n-2) \int_{M_{0}} \kappa\left([d \theta]^{2}[\widetilde{\omega}]^{n-3}\right) \cdot \int_{M_{0}} \kappa\left([\widetilde{\omega}]^{n-1}\right)-(n-1)\left(\int_{M_{0}} \kappa\left([d \theta][\widetilde{\omega}]^{n-2}\right)\right)^{2} \leq 0 .
$$

To compute the above integrals $\int_{M_{0}} \kappa\left([d \theta]^{2}[\widetilde{\omega}]^{n-3}\right), \int_{M_{0}} \kappa\left([\widetilde{\omega}]^{n-1}\right)$, and $\int_{M_{0}}$ $\kappa\left([d \theta][\widetilde{\omega}]^{n-2}\right)$, we need the residue formula due to Jeffrey and Kirwan. (See [10] and $[9]$.)

Theorem 3.7 ([10]). Let $\nu \in H_{S^{1}}^{*}(M ; \mathbb{R})$. Then

$$
\int_{M_{0}} \kappa(\nu)=\sum_{F \in M^{S^{1}}, \mu(F)>0} \operatorname{Res}_{u=0}\left(\frac{\left.\nu\right|_{F}}{e_{F}}\right) .
$$

Here, $e_{F}$ is the equivariant Euler class of the normal bundle to $F$ so that we can regard $\left.\nu\right|_{F}$ and $e_{F}$ as polynomials with one variable $u$. $\operatorname{Res}_{u=0}(f)$ means a residue of $f$ where $f$ is a rational function with one variable $u$.

Now, let's compute $\int_{M_{0}} \kappa\left([d \theta]^{2}[\widetilde{\omega}]^{n-3}\right)$. By Theorem 3.7 .

$$
\int_{M_{0}} \kappa\left([d \theta]^{2}[\widetilde{\omega}]^{n-3}\right)=\sum_{F \in M^{S^{1}}, \mu(F)>0} \operatorname{Res}_{u=0}\left(\frac{\left.[d \theta]^{2}[\widetilde{\omega}]^{n-3}\right|_{F}}{e_{F}}\right) .
$$

Since $\left.[\widetilde{\omega}]\right|_{z}=\mu(z) u$ and $[d \theta] \mid z=-u$ by Lemma 3.4 and 3.5. we have

$$
\begin{aligned}
\int_{M_{0}} \kappa\left([d \theta]^{2}[\widetilde{\omega}]^{n-3}\right) & =\sum_{F \in M^{S^{1}}, \mu(F)>0} \operatorname{Res}_{u=0}\left(\frac{\mu(F)^{n-3} u^{n-1}}{e_{F}}\right) \\
& =\sum_{F \in M^{S^{1}}, \mu(F)>0} \operatorname{Res}_{u=0}\left(\frac{\mu(F)^{n-3} u^{n-1}}{m_{F} u^{n}}\right) \\
& =\sum_{F \in M^{S^{1}}, \mu(F)>0} \frac{1}{m_{F}} \mu(F)^{n-3},
\end{aligned}
$$


where $m_{F}$ is the product of all weights of tangential $S^{1}$-representation at $F$. Similarly, if $\xi \in \mathbb{R}$ is a regular value of $\mu$, then we let $\widetilde{\mu}=\mu-\xi$ be the new moment map. By the same argument, we have the following lemma.

Lemma 3.8. For a regular value $\xi$ of the moment map $\mu$, we have the following:

$$
\begin{gathered}
\int_{M_{0}} \kappa\left([d \theta]^{2}[\widetilde{\omega}]^{n-3}\right)=\sum_{\substack{F \in M^{1} \\
\mu(F)>\xi^{\prime}}} \frac{1}{m_{F}}(\mu(F)-\xi)^{n-3} . \\
\int_{M_{0}} \kappa\left([d \theta][\widetilde{\omega}]^{n-2}\right)=\sum_{\substack{F \in M S^{1} \\
\mu(F)>\xi^{\prime}}} \frac{-1}{m_{F}}(\mu(F)-\xi)^{n-2} .
\end{gathered}
$$

$$
\int_{M_{0}} \kappa\left([\widetilde{\omega}]^{n-1}\right)=\sum_{\substack{F \in M^{1} \\ \mu(F)>\xi}} \frac{1}{m_{F}}(\mu(F)-\xi)^{n-1} .
$$

Combining Corollary 3.6 and Lemma 3.8, we have the following proposition.

Proposition 3.9. Let $(M, \omega)$ be a closed Hamiltonian $S^{1}$-manifold with the moment map $\mu: M \rightarrow \mathbb{R}$. Assume that $M^{S^{1}}$ consists of isolated fixed points. Then a density function of the Duistermaat-Heckman measure with respect to $\mu$ is logconcave if and only if

$$
\sum_{\substack{F \in M S^{1} \\ \mu(F)>\xi}} \frac{1}{m_{F}}(\mu(F)-\xi)^{n-3} \cdot \sum_{\substack{F \in M^{1} \\ \mu(F)>\xi}} \frac{1}{m_{F}}(\mu(F)-\xi)^{n-1}-\left(\sum_{\substack{F \in M S^{1} \\ \mu(F)>\xi}} \frac{1}{m_{F}}(\mu(F)-\xi)^{n-2}\right)^{2} \leq 0
$$

for every regular value $\xi \in \mu(M)$, where $m_{F}$ is the product of all weights of the $S^{1}$-representation on $T_{F} M$. In particular, the log-concavity of the DuistermaatHeckman measure is completely determined by the set $\left\{\left(\mu(F), m_{F}\right)_{F} \mid F \in M^{S^{1}}\right\}$.

Corollary 3.10. Let $\left(M^{2 n}, \omega\right)$ and $\left(N^{2 n}, \sigma\right)$ be two closed Hamiltonian $S^{1}$ manifolds with the moment maps $\mu_{1}$ and $\mu_{2}$, respectively. Assume there exists a bijection $\phi: M^{S^{1}} \rightarrow N^{S^{1}}$ which satisfies

(1) for each $F \in M^{S^{1}}, m_{F}=m_{\phi(F)}$, and

(2) for each $F \in M^{S^{1}}, \mu_{1}(F)=\mu_{2}(\phi(F))$,

where $m_{F}$ is the product of all weights of the tangential $S^{1}$-representation at $F$. If $N$ satisfies the log-concavity of the Duistermaat-Heckman measure with respect to $\mu_{2}$, then so does $M$ with respect to $\mu_{1}$.

Remark 3.11. The integration formulae (1) and (3) in Lemma 3.8 are proved by Wu by using the stationary phase method. See Theorem 5.2 in [21] for the details.

\section{Proof of Theorem 1.1}

As noted in the introduction, if a Hamiltonian $S^{1}$-action on the Kähler manifold is holomorphic, then the corresponding Duistermaat-Heckman function is logconcave by [7]. Let $\left(M^{2 n}, \omega\right)$ be a closed semifree Hamiltonian $S^{1}$-manifold with the moment map $\mu$. Assume that all fixed points are isolated. Let DH be the corresponding Duistermaat-Heckman function with respect to $\mu$. We will show that there 
is a Kähler form $\omega_{1} \oplus \cdots \oplus \omega_{n}$ on $S^{2} \times \cdots \times S^{2}$ with the standard diagonal holomorphic semifree circle action such that a bijection $\psi^{-1} \circ \phi: M^{S^{1}} \rightarrow\left(S^{2} \times \cdots \times S^{2}\right)^{S^{1}}$ given in Section 2 satisfies the conditions in Corollary 3.10, which implies the logconcavity of DH. Now we start with the lemma below.

Lemma 4.1. Let $\left(M^{2 n}, \omega\right)$ be a closed semifree Hamiltonian circle action with the moment map $\mu$. Assume that all fixed points are isolated. Then $\left\{\left(\mu(F), m_{F}\right)_{F} \mid F \in\right.$ $\left.M^{S^{1}}\right\}$ is completely determined by $\mu\left(p_{0}^{1}\right), \mu\left(p_{1}^{1}\right), \cdots, \mu\left(p_{1}^{n}\right)$, where the $p_{k}^{j}$ 's are the fixed points of index $2 k$ for $j=1, \cdots,\left(\begin{array}{l}n \\ k\end{array}\right)$.

Proof. Consider an equivariant symplectic 2-form $\widetilde{\omega}$ on $M \times{ }_{S^{1}} E S^{1}$ which is given in Section 3. Since the set $\left\{x, a_{1}, \cdots, a_{n}\right\}$ is a basis of $H_{S^{1}}^{2}(M ; \mathbb{Z})$, we may assume

$$
[\widetilde{\omega}]=m_{0} x+m_{1} a_{1}+\cdots+m_{n} a_{n}
$$

for some real numbers $m_{i}$. (See Section 2, the definition of $x, a_{1}, \cdots, a_{n}$.) Therefore, for any fixed point $p_{i}^{j} \in M^{S^{1}}$, we have

$$
\left.[\widetilde{\omega}]\right|_{p_{i}^{j}}=\left.\left(m_{0} x+m_{1} a_{1}+\cdots+m_{n} a_{n}\right)\right|_{p_{i}^{j}} .
$$

When $i=0$ and $j=1$, Lemma 3.4 implies that

$$
\left.[\widetilde{\omega}]\right|_{p_{0}^{1}}=m_{0} u .
$$

Since every $a_{i}$ vanishes on $p_{0}^{1}$, the right hand side is

$$
\left.\left(m_{0} x+m_{1} a_{1}+\cdots+m_{n} a_{n}\right)\right|_{p_{0}^{1}}=m_{0} u .
$$

Hence $m_{0}=\mu\left(p_{0}^{1}\right)$. Similarly,

$$
\left.[\widetilde{\omega}]\right|_{p_{1}^{i}}=\mu\left(p_{1}^{i}\right) u
$$

and

$$
\left.\left(m_{0} x+m_{1} a_{1}+\cdots+m_{n} a_{n}\right)\right|_{p_{1}^{i}}=m_{0} u+m_{i} u .
$$

Hence we have $m_{i}=\mu\left(p_{1}^{i}\right)-m_{0}=\mu\left(p_{1}^{i}\right)-\mu\left(p_{0}^{1}\right)$ for each $i=1, \cdots, n$. Therefore $\left\{\mu\left(p_{0}^{1}\right), \mu\left(p_{1}^{1}\right), \cdots, \mu\left(p_{1}^{n}\right)\right\}$ determines the coefficients $m_{i}$ of $[\widetilde{\omega}]$.

For $p_{k}^{j}$ with $k>1$, the relation $\left.[\widetilde{\omega}]\right|_{p_{k}^{j}}=\left.\left(m_{0} x+m_{1} a_{1}+\cdots+m_{n} a_{n}\right)\right|_{p_{k}^{j}}$ implies

- $\left.[\widetilde{\omega}]\right|_{p_{k}^{j}}=\mu\left(p_{k}^{j}\right) u$ and

- $\left.\left(m_{0} x+m_{1} a_{1}+\cdots+m_{n} a_{n}\right)\right|_{p_{k}^{j}}=m_{0} u+\sum_{i \in \phi\left(p_{k}^{j}\right)} m_{i} u$.

Therefore, for fixed $k$, the set $\left\{\left(\mu\left(p_{k}^{j}\right), m_{p_{k}^{j}}\right) j \mid j=1, \cdots,\left(\begin{array}{l}n \\ k\end{array}\right)\right\}$ is just

$$
\left\{\left(\left(m_{0}+m_{i_{1}}+\cdots+m_{i_{k}}\right),(-1)^{k}\right)_{\left\{i_{1}, \cdots, i_{k}\right\}} \mid\left\{i_{1}, \cdots, i_{k}\right\} \subset\{1,2, \cdots, n\}\right\},
$$

and this set does not depend on the ordering of $p_{k}^{j}$. Hence $\left\{\left(\mu(F), m_{F}\right)_{F} \mid F \in\right.$ $\left.M^{S^{1}}\right\}=\bigcup_{k=0}^{k=n}\left\{\left(\mu\left(p_{k}^{j}\right), m_{p_{k}^{j}}\right)_{j} \mid j=1, \cdots,\left(\begin{array}{l}n \\ k\end{array}\right)\right\}$ is completely determined by $\mu\left(p_{0}^{1}\right)$, $\mu\left(p_{1}^{1}\right), \cdots, \mu\left(p_{1}^{n}\right)$.

Now we are ready to prove Theorem 1.1 .

Theorem 4.2 (Theorem 1.1). Let $(M, \omega)$ be a closed symplectic manifold with a semifree Hamiltonian $S^{1}$-action whose fixed point set $M^{S^{1}}$ consists of isolated points. Then the Duistermaat-Heckman measure is log-concave. 
Proof. Let $\mu: M \rightarrow \mathbb{R}$ be a moment map and let $M^{S^{1}}=\left\{p_{k}^{j} \mid k=0, \cdots, n, j=\right.$ $\left.1, \cdots,\left(\begin{array}{l}n \\ k\end{array}\right)\right\}$ be the fixed point set, where $p_{k}^{j}$ is a fixed point of index $2 k$ labeled by $j=1, \cdots,\left(\begin{array}{l}n \\ k\end{array}\right)$. Note that $\mu\left(p_{0}^{1}\right)$ is the minimum value of $\mu$. Let $\phi: M^{S^{1}} \rightarrow 2^{[n]}$ be the identification map between the fixed point set $M^{S^{1}}$ and the power set $2^{[n]}$ defined in Theorem 2.2. Then we may assume (by re-labeling, if necessary) that $\phi\left(p_{1}^{j}\right)=\{j\}$ for $j=1, \cdots, n$. We will show that there exists a semifree holomorphic Hamiltonian $S^{1}$-manifold $\left.\left(S^{2} \times \cdots \times S^{2}\right), \sigma\right)$ with the moment map $\mu^{\prime}$ such that $\psi^{-1} \circ \phi: M^{S^{1}} \rightarrow\left(S^{2} \times \cdots \times S^{2}\right)^{S^{1}}$ preserves their indices, weights, and the values of the moment map, where $\psi:\left(S^{2} \times \cdots \times S^{2}\right)^{S^{1}} \rightarrow 2^{[n]}$ is the identification map described in Theorem 2.2 .

Let $\omega_{i}$ be the Fubini-Study form on $S^{2}$ such that the symplectic volume is $\mu\left(p_{1}^{i}\right)-$ $\mu\left(p_{0}^{1}\right)$. Let $S$ be the south pole and $N$ be the north pole of $S^{2}$ so that $S$ is the minimum ( $N$ is the maximum, respectively) of the moment map on $\left(S^{2}, \omega_{i}\right)$ with the standard semifree circle action on $S^{2}$. Then $\left(S^{2} \times \cdots \times S^{2}, \omega_{1} \oplus \cdots \oplus \omega_{n}\right)$ is a symplectic manifold with the diagonal semifree Hamiltonian circle action. Let $\mu^{\prime}: S^{2} \times \cdots \times S^{2} \rightarrow \mathbb{R}$ be the moment map whose minimum is $\mu\left(p_{0}^{1}\right)$. Let $\psi$ : $\left(S^{2} \times \cdots \times S^{2}\right)^{S^{1}} \rightarrow 2^{[n]}$ be the identification map between the fixed point set $\left(S^{2} \times \cdots \times S^{2}\right)^{S^{1}}$ and the power set $2^{[n]}$ such that

$$
\psi^{-1}(\{i\}):=q_{1}^{i}=(S, \cdots, S, N, S, \cdots, S)
$$

for all $i=1, \cdots, n$, where $q_{1}^{i}=(S, \cdots, S, N, S, \cdots, S)$ is a fixed point on $\left(S^{2} \times\right.$ $\left.\cdots \times S^{2}\right)$ of index 2 such that the $i$-th coordinate is $N$ and the other coordinates are $S$. Then we can easily see that $\psi^{-1} \circ \phi\left(p_{1}^{j}\right)=q_{1}^{j}$ and $\mu\left(p_{1}^{j}\right)=\mu^{\prime}\left(q_{1}^{j}\right)=\mu\left(p_{1}^{j}\right)-\mu\left(p_{0}^{1}\right)$ for all $j=1, \cdots, n$. By Lemma 4.1, we have

$$
\left\{\left(\mu(F), m_{F}\right)_{F} \mid F \in M^{S^{1}}\right\}=\left\{\left(\mu^{\prime}(F), m_{F}\right)_{F} \mid F \in\left(S^{2} \times \cdots \times S^{2}\right)^{S^{1}}\right\},
$$

and $\psi^{-1} \circ \phi: M^{S^{1}} \rightarrow\left(S^{2} \times \cdots \times S^{2}\right)^{S^{1}}$ satisfies the condition in Corollary 3.10, Therefore the Duistermaat-Heckman measure is log-concave on $\mu(M)$.

Remark 4.3 (Summary). Let $(M, \omega)$ be a $2 n$-dimensional compact semifree Hamiltonian $S^{1}$-manifold with isolated fixed points. Let $\mu: M \rightarrow \mathbb{R}$ be a moment map. Let $\phi: M^{S^{1}} \rightarrow 2^{[n]}$ be the identification described in Theorem 2.2, In the proof of Theorem 1.1. we proved that there exists a Kähler form $\omega_{1} \oplus \cdots \oplus \omega_{n}$ on $S^{2} \times \cdots \times S^{2}$ with the diagonal semifree Hamiltonian action with the moment map $\mu^{\prime}: S^{2} \times \cdots \times S^{2} \rightarrow \mathbb{R}$ satisfying the following:

- There is an identification $\psi:\left(S^{2} \times \cdots \times S^{2}\right)^{S^{1}} \rightarrow 2^{[n]}$ such that $\psi^{-1}(\{i\})=$ $q_{1}^{i}=(S, \cdots, S, N, S, \cdots, S)$, where $q_{1}^{i}=(S, \cdots, S, N, S, \cdots, S)$ is a fixed point on $S^{2} \times \cdots \times S^{2}$ of index 2 such that the $i$-th coordinate is $N$ and the other coordinates are $S$.

- The composition map $\psi^{-1} \circ \phi: M^{S^{1}} \rightarrow\left(S^{2} \times \cdots \times S^{2}\right)^{S^{1}}$ preserves their indices, weights, and the values of the moment map.

- By Corollary 2.4, $\psi^{-1} \cdots \phi: M^{S^{1}} \rightarrow\left(S^{2} \times \cdots \times S^{2}\right)^{S^{1}}$ induces an isomorphism $f: H_{S^{1}}^{*}(M ; \mathbb{Z}) \rightarrow H_{S^{1}}^{*}\left(S^{2} \times \cdots \times S^{2} ; \mathbb{Z}\right)$. Moreover, by the proof of Lemma 4.1] $f$ sends the equivariant symplectic class $[\widetilde{\omega}]$ in $H_{S^{1}}^{2}(M ; \mathbb{R})$ to the one in $H_{S^{1}}^{2}\left(S^{2} \times \cdots \times S^{2} ; \mathbb{R}\right)$.

We will refer to Remark 4.3 in the proof of Theorem 1.2 in Section 5 . 


\section{The HARD LeFsCHETZ PROPERTY \\ OF THE REDUCED SYMPLECTIC FORMS}

For a Kähler manifold $(N, \sigma)$ with a holomorphic circle action preserving $\sigma$, let $t \in R$ be any regular value of the moment map $H: N \rightarrow \mathbb{R}$. Since the reduced space $N_{t}:=H^{-1}(t) / S^{1}$ with the reduced symplectic form $\sigma_{t}$ is again Kähler, $\sigma_{t}$ satisfies the hard Lefschetz property for every regular value $t \in \mathbb{R}$. In this section, we show that the same thing happens when $(M, \omega)$ is a closed semifree Hamiltonian $S^{1}$-manifold whose fixed points are all isolated. The following theorem is due to Tolman and Weitsman.

Theorem $5.1([20])$. Let $(M, \omega)$ be a closed Hamiltonian $S^{1}$-manifold with a moment map $\mu: M \rightarrow \mathbb{R}$. Assume that all fixed points are isolated and 0 is a regular value. Let $M^{S^{1}}$ be the set of fixed points. Define $K_{+}^{M}:=\left\{\alpha \in H_{S^{1}}^{*}(M ; \mathbb{Z})|\alpha|_{F_{+}}=\right.$ $0\}$ where $F_{+}:=M^{S^{1}} \cap \mu^{-1}(0, \infty)$ and $K_{-}^{M}:=\left\{\alpha \in H_{S^{1}}^{*}(M ; \mathbb{Z})|\alpha|_{F_{-}}=0\right\}$ where $F_{-}:=M^{S^{1}} \cap \mu^{-1}(-\infty, 0)$. Then there is a short exact sequence

$$
0 \longrightarrow K \longrightarrow H_{S^{1}}^{*}(M ; \mathbb{Z}) \stackrel{\kappa}{\longrightarrow}\left(M_{\text {red }} ; \mathbb{Z}\right) \longrightarrow 0
$$

where $\kappa$ is the Kirwan map.

Now we are ready to prove Theorem 1.2 ,

Theorem 5.2 (Theorem 1.2). Let $(M, \omega)$ be a closed semifree Hamiltonian $S^{1}$ manifold whose fixed points are all isolated, and let $\mu$ be the moment map. Then $\omega$ satisfies the hard Lefschetz property. Moreover, the reduced symplectic form $\omega_{t}$ satisfies the hard Lefschetz property for every regular value $t$.

Proof. Let $\mu: M \rightarrow \mathbb{R}$ be a moment map such that $0 \in \mathbb{R}$ is a regular value of $\mu$. For $M_{\text {red }} \cong \mu^{-1}(0) / S^{1}$ with the reduced symplectic form $\omega_{0}$, let $\kappa_{M}: H_{S^{1}}^{*}(M ; \mathbb{R}) \rightarrow$ $H^{*}\left(M_{\text {red }} ; \mathbb{R}\right)$ be the Kirwan map for $(M, \omega)$ and let $\kappa$ be the one for $\left(S^{2} \times \cdots \times S^{2}, \sigma\right)$, where $\sigma:=\omega_{1} \oplus \cdots \oplus \omega_{n}$ is chosen in the proof of Theorem 1.1 in Section 4. (See also Remark 4.3.) As in Remark 4.3, we proved that there exists a semifree holomorphic Hamiltonian $S^{1}$-manifold $\left(S^{2} \times \cdots \times S^{2}, \sigma\right)$ with the moment map $\mu^{\prime}$ such that $\psi^{-1} \circ \phi: M^{S^{1}} \rightarrow\left(S^{2} \times \cdots \times S^{2}\right)^{S^{1}}$ preserves their indices, weights, and the values of the moment map. Also, the induced ring isomorphism $f: H_{S^{1}}^{*}(M ; \mathbb{Z}) \rightarrow$ $H_{S^{1}}^{*}\left(S^{2} \times \cdots \times S^{2} ; \mathbb{Z}\right)$ given in Corollary 2.4 satisfies $\left.\alpha\right|_{x}=\left.f(\alpha)\right|_{\psi^{-1} \circ \phi(x)}$ for any $\alpha \in H_{S^{1}}^{*}(M ; \mathbb{Z})$ and any fixed point $x \in M^{S^{1}}$. Hence $\psi^{-1} \circ \phi$ identifies $K_{+}^{M}$ with $K_{+}^{S 2 \times \cdots \times S^{2}}$ and $K_{-}^{M}$ with $K_{-}^{S 2 \times \cdots \times S^{2}}$. Hence if $\alpha \in K_{+}^{M}$, then $f(\alpha) \in K_{+}^{S^{2} \times \cdots \times S^{2}}$. Similarly for any $\alpha \in K_{-}^{M}$, we have $f(\alpha) \in K_{-}^{S^{2} \times \cdots \times S^{2}}$. Therefore $f(\alpha)$ is in ker $\kappa$ if and only if $\alpha \in \operatorname{ker} \kappa_{M}$ by Theorem 5.1

Now, let $\widetilde{\omega}$ be the equivariant symplectic form with respect to the moment map $\mu$. (See Section [3.) Note that $\kappa(f([\widetilde{\omega}]))$ is the cohomology class of the reduced symplectic form of $S^{2} \times \cdots \times S^{2}$ at $\mu^{-1}(0) / S^{1}$. Since the Kähler quotient of the holomorphic action is again Kähler, $\kappa(f([\widetilde{\omega}]))$ satisfies the hard Lefschetz property. Now, assume that $\omega_{0}$ does not satisfy the hard Lefschetz property. Then there exists a positive integer $k(<n)$ and some non-zero $\alpha \in H^{k}\left(M_{\text {red }} ; \mathbb{R}\right)$ such that $\alpha \cdot\left[\omega_{0}\right]^{n-k}=0$ in $H^{2 n-k}\left(M_{\text {red }}\right)$. By the Kirwan surjectivity theorem [13, we can find $\widetilde{\alpha} \in H_{S^{1}}^{k}(M ; \mathbb{R})$ with $\kappa(\widetilde{\alpha})=\alpha$. Then $\widetilde{\alpha} \cdot[\widetilde{\omega}]^{n-k}$ is in ker $\kappa_{M}$ and hence the image $f\left(\widetilde{\alpha} \cdot[\widetilde{\omega}]^{n-k}\right)$ is in ker $\kappa$. It implies that $f(\widetilde{\alpha})=0$ by the hard Lefschetz 
condition for $f([\widetilde{\omega}])$. Since $[\widetilde{\alpha}] \in \operatorname{ker} \kappa_{M}$ if and only if $f([\widetilde{\alpha}]) \in \operatorname{ker} \kappa$ and $[\widetilde{\alpha}]$ is not in ker $\kappa_{M}$, it is a contradiction.

It remains to show that $(M, \omega)$ satisfies the hard Lefschetz property. Recall that $\psi^{-1} \circ \phi: M^{S^{1}} \rightarrow\left(S^{2} \times \cdots \times S^{2}\right)^{S^{1}}$ induces an isomorphism

$$
f: H_{S^{1}}^{*}(M ; \mathbb{Z}) \rightarrow H_{S^{1}}^{*}\left(S^{2} \times \cdots \times S^{2} ; \mathbb{Z}\right),
$$

which sends the equivariant symplectic class $[\widetilde{\omega}]$ to $[\widetilde{\sigma}]$ as we have seen in Section 4 . (See Remark 4.3.) Here, $\widetilde{\sigma}$ is an equivariant symplectic form induced by $\sigma-d\left(\mu^{\prime} \theta\right)$ in $H_{S^{1}}^{*}\left(S^{2} \times \cdots \times S^{2} ; \mathbb{R}\right)$. Since $f$ is an $H^{*}\left(B S^{1} ; \mathbb{R}\right)$-algebra isomorphism, it induces a ring isomorphism

$$
f_{u}: \frac{H_{S^{1}}^{*}(M ; \mathbb{R})}{u \cdot H_{S^{1}}^{*}(M ; \mathbb{R})} \rightarrow \frac{H_{S^{1}}^{*}\left(S^{2} \times \cdots \times S^{2} ; \mathbb{R}\right)}{u \cdot H_{S^{1}}^{*}\left(S^{2} \times \cdots \times S^{2} ; \mathbb{R}\right)}
$$

Moreover, the quotient map $\pi_{M}: H_{S^{1}}^{*}(M ; \mathbb{R}) \rightarrow \frac{H_{S^{1}}^{*}(M ; \mathbb{R})}{u \cdot H_{S^{1}}^{*}(M ; \mathbb{R})} \cong H^{*}(M ; \mathbb{R})\left(\pi_{S^{2} \times \cdots \times S^{2}}\right.$, resp.) is a ring homomorphism which comes from an inclusion $M \hookrightarrow M \times{ }_{S^{1}} E S^{1}$ as a fiber. Therefore $\pi_{M}([\widetilde{\omega}])=[\omega]$ and $\pi_{S^{2} \times \cdots \times S^{2}}([\widetilde{\sigma}])=[\sigma]$. Therefore the isomorphism $f_{u}$ maps $[\omega]$ to $[\sigma]$. Since $\sigma$ is a Kähler form, it satisfies the hard Lefschetz property. Hence so does $\omega$.

\section{REFERENCES}

[1] Michèle Audin, Torus actions on symplectic manifolds, Second revised edition, Progress in Mathematics, vol. 93, Birkhäuser Verlag, Basel, 2004. MR2091310 (2005k:53158)

[2] Yunhyung Cho and Min Kyu Kim, Log-concavity of complexity one Hamiltonian torus actions (English, with English and French summaries), C. R. Math. Acad. Sci. Paris 350 (2012), no. 17-18, 845-848, DOI 10.1016/j.crma.2012.07.004. MR2989389

[3] Y. Cho, T. Hwang and D. Y. Suh, Semifree Hamiltonian circle actions on 6-dimensional symplectic manifolds with non-isolated fixed point set, arXiv:1005.0193 (2010).

[4] Thomas Delzant, Hamiltoniens périodiques et images convexes de l'application moment (French, with English summary), Bull. Soc. Math. France 116 (1988), no. 3, 315-339. MR984900 (90b:58069)

[5] J. J. Duistermaat and G. J. Heckman, On the variation in the cohomology of the symplectic form of the reduced phase space, Invent. Math. 69 (1982), no. 2, 259-268, DOI 10.1007/BF01399506. MR674406 (84h:58051a)

[6] Eduardo González, Classifying semi-free Hamiltonian $S^{1}$-manifolds, Int. Math. Res. Not. IMRN 2 (2011), 387-418. MR2764868 (2012g:53177)

[7] William Graham, Logarithmic convexity of push-forward measures, Invent. Math. 123 (1996), no. 2, 315-322, DOI 10.1007/s002220050029. MR1374203 (96m:58081)

[8] V. Guillemin, E. Lerman, and S. Sternberg, On the Kostant multiplicity formula, J. Geom. Phys. 5 (1988), no. 4, 721-750 (1989), DOI 10.1016/0393-0440(88)90026-5. MR.1075729 (92f:58058)

[9] Lisa C. Jeffrey, The residue formula and the Tolman-Weitsman theorem, J. Reine Angew. Math. 562 (2003), 51-58, DOI 10.1515/crll.2003.077. MR2011331(2005b:53135)

[10] Lisa C. Jeffrey and Frances C. Kirwan, Localization for nonabelian group actions, Topology 34 (1995), no. 2, 291-327, DOI 10.1016/0040-9383(94)00028-J. MR1318878 (97g:58057)

[11] Yael Karshon, Example of a non-log-concave Duistermaat-Heckman measure, Math. Res. Lett. 3 (1996), no. 4, 537-540. MR1406018 (97f:58056)

[12] Yael Karshon, Periodic Hamiltonian flows on four-dimensional manifolds, Mem. Amer. Math. Soc. 141 (1999), no. 672, viii+71. MR.1612833 (2000c:53113)

[13] Frances Clare Kirwan, Cohomology of quotients in symplectic and algebraic geometry, Mathematical Notes, vol. 31, Princeton University Press, Princeton, NJ, 1984. MR766741 (86i:58050)

[14] Yi Lin, The log-concavity conjecture for the Duistermaat-Heckman measure revisited, Int. Math. Res. Not. IMRN 10 (2008), Art. ID rnn027, 19, DOI 10.1093/imrn/rnn027. MR2429245 (2009i:53086) 
[15] Yi Lin, Examples of non-Kähler Hamiltonian circle manifolds with the strong Lefschetz property, Adv. Math. 208 (2007), no. 2, 699-709, DOI 10.1016/j.aim.2006.03.011. MR2304334 (2008h:53149)

[16] Andrei Okounkov, Log-concavity of multiplicities with application to characters of $\mathrm{U}(\infty)$, Adv. Math. 127 (1997), no. 2, 258-282, DOI 10.1006/aima.1997.1622. MR.1448715 (99a:22028)

[17] Andrei Okounkov, Brunn-Minkowski inequality for multiplicities, Invent. Math. 125 (1996), no. 3, 405-411, DOI 10.1007/s002220050081. MR.1400312 (99a:58074)

[18] Andrei Okounkov, Why would multiplicities be log-concave?, The orbit method in geometry and physics (Marseille, 2000), Progr. Math., vol. 213, Birkhäuser Boston, Boston, MA, 2003, pp. 329-347. MR1995384(2004j:20022)

[19] Susan Tolman and Jonathan Weitsman, On semifree symplectic circle actions with isolated fixed points, Topology 39 (2000), no. 2, 299-309, DOI 10.1016/S0040-9383(99)00011-7. MR1722020 (2000k:53074)

[20] Susan Tolman and Jonathan Weitsman, The cohomology rings of symplectic quotients, Comm. Anal. Geom. 11 (2003), no. 4, 751-773. MR2015175 (2004k:53140)

[21] Siye Wu, An integration formula for the square of moment maps of circle actions, Lett. Math. Phys. 29 (1993), no. 4, 311-328, DOI 10.1007/BF00750965. MR1257832 (95b:58070)

School of Mathematics, Korea Institute for Advanced Study (KIAS), 87 Hoegiro, Dongdaemun-Gu, Seoul, 130-722, Republic of Korea

E-mail address: yhcho@kias.re.kr 